\title{
Mechanical saturation of common architectural coated fabrics
}

\author{
J. Uhlemann*, N. Stranghöner*, M. Motevalli ${ }^{\dagger}$ and D. Balzani ${ }^{\dagger}$ \\ ${ }^{*}$ University of Duisburg-Essen, Institute for Metal and Lightweight Structures, Universitaetsstr. 15, \\ 45141 Essen, Germany \\ Email: joerg.uhlemann@uni-due.de, natalie.stranghoener@uni-due.de \\ Web page: www.uni-due.de/iml \\ ${ }^{\dagger}$ Ruhr University Bochum, Chair of Continuum Mechanics, Universitaetsstr. 150, \\ 44801 Bochum, Germany \\ Email:mehran.motevalli@rub.de,daniel.balzani@rub.de \\ Web page: www.lkm.rub.de
}

\begin{abstract}
Architectural coated fabrics, which are mainly woven textiles made from polyester or glass fibre yarns, are known to exhibit a nonlinear and viscoplastic stress-strain behaviour. The behaviour changes with the number of load cycles applied. Changes concern global slope and degree of nonlinearity of stressstrain paths and the magnitude of remaining irreversible strain after unloading. These changes are huge in the first load cycles and decline rapidly in the following ones. This shows an increasing mechanical saturation to a mainly elastic behaviour. However, some of the changes of the mechanical properties do not come to a halt. Only in infinity they approach an invariable, mechanically saturated state. Nonetheless, in a state acceptably close to the ideal saturated state, the stress-strain paths reveal the elastic share of the initially inelastic stress-strain paths of woven fabrics.

This contribution investigates the number of load cycles required to achieve a mechanically saturated state by means of monotonous cyclic biaxial tensile tests on PVC-coated polyester fabrics and PTFEcoated glass fibre fabrics. It is found that irreversible strain increments decrease fast to low constant levels with low scatter after approximately a dozen load cycles. In contrast to that, other properties like the global slope or nonlinearity of the stress-strain paths saturate only after a number of load cycles of several orders of magnitude more than those for the irreversible strain. Changes of stiffness parameters in late cycles are slow but can be considerably compared to early ones. For instance, the elastic moduli in load cycle 1000 can be roughly twice as high as the ones of load cycle 5 .

This paper provides an extrapolation method to estimate the late load cycle behaviour from early cycle test data and puts the findings in context to material testing, structural analysis and future developments of material modelling.
\end{abstract}

